\title{
Two-Dimensional Densely Packed DNA Nanostructure Derived from DNA Complexation with Low-Generation Poly(amidoamine) Dendrimer
}

\author{
Chun-Jen $\mathrm{Su}^{1}$, Yi-Chun Liu ${ }^{2}$, Hsin-Lung Chen ${ }^{1 *}$, Yen-Cheng Li $^{3}$, Hsien-Kuang Lin ${ }^{2}$, Wen-Liang \\ $\mathrm{Liu}^{2}$ and Chain-Shu $\mathrm{Hsu}^{3}$ \\ ${ }^{1}$ Department of Chemical Engineering, National Tsing Hua University, Hsin-Chu 30013, Taiwan \\ ${ }^{2}$ Materials Research Laboratories, Industrial Technology Research Institute, Chutung, Hsin-Chu, \\ Taiwan
}

${ }^{3}$ Department of Applied Chemistry, National Chiao Tung University, Hsin-Chu 30050, Taiwan

\section{Supporting Information}

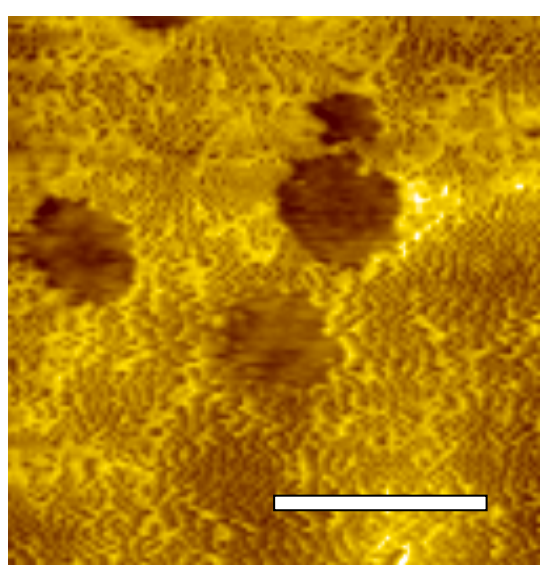

(a)

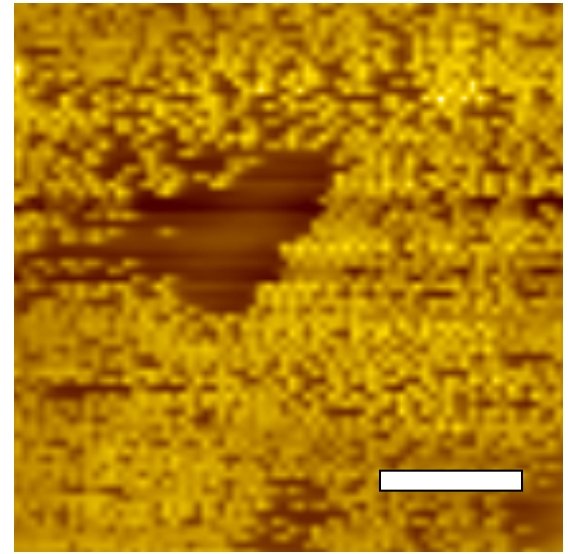

(b)

Figure 1S Additional AFM topographic images showing the formation of 2-D ordered DNA arrays on mica surface at different places in the same sample as that used for obtaining the image in Figure 3. The scale bar corresponds to $1 \mu \mathrm{m}$ and $20 \mathrm{~nm}$ in (a) and (b), respectively.. 